Cahiers
de la Recherche
Furles Droits

Cahiers de la recherche sur les droits fondamentaux

$4 \mid 2005$

Quel avenir pour la laïcité cent ans après la loi de 1905 ?

\title{
L'hôpital, le patient, le juge et la religion
}

Juge des référés du Tribunal administratif de Caen, ordonnance du 26 avril 2005, Association locale pour le culte des témoins de Jéhovah de Lisieux et M. François D c / Hôpital local d'Orbec-en-Auge, requête $\mathrm{n}^{\circ}$ 0500914, M. Heu, président, $\mathrm{M}^{\text {mes }}$ Trizac et Rayssac, avocats

\section{Xavier Mondésert}

\section{(2) OpenEdition}

\section{Journals}

Édition électronique

URL : https://journals.openedition.org/crdf/7293

DOI : $10.4000 /$ crdf.7293

ISSN : 2264-1246

Éditeur

Presses universitaires de Caen

Édition imprimée

Date de publication : 1 décembre 2005

Pagination : 79-86

ISBN : 2-84133-250-0

ISSN : $1634-8842$

Référence électronique

Xavier Mondésert, «L'hôpital, le patient, le juge et la religion », Cahiers de la recherche sur les droits fondamentaux [En ligne], 4 | 2005, mis en ligne le 15 décembre 2020, consulté le 17 novembre 2022 URL : http://journals.openedition.org/crdf/7293 ; DOI : https://doi.org/10.4000/crdf.7293 


\title{
L'hôpital, le patient, le juge et la religion
}

\author{
Juge des référés du Tribunal administratif de Caen, \\ ordonnance du 26 avril 2005, \\ Association locale pour le culte des témoins de Jéhovah de Lisieux et $M$. François $D$ \\ c / Hôpital local d'Orbec-en-Auge, requête n ${ }^{0} 0500914$, \\ M. Heu, président, $\mathrm{M}^{\text {mes }}$ Trizac et Rayssac, avocats
}

\author{
I. Sur les fins de non-recevoir soulevées par l'hôpital local d'Orbec \\ II. Sur les conclusions aux fins de suspension \\ A. En ce qui concerne la condition d'urgence \\ B. En ce qui concerne la condition tenant à l'existence d'un doute sérieux \\ III. Sur les conclusions tendant à l'application de l'article L. 761-1 du code de justice administrative \\ IV. La maladresse de l'administration \\ V. L'ambiguïté du juge administratif
}

Vu la requête et le mémoire complémentaire, enregistrés respectivement les 15 et 21 avril 2005 au greffe du Tribunal administratif de Caen, sous le nº 0500914, présentés pour l'Association locale pour le culte des témoins de Jéhovah de Lisieux, dont le siège est situé aux Bruyères, Saint-Désir (14100), et M. François D, demeurant à Lisieux (14100), par Maître Michel Trizac, avocat.

L’Association locale pour le culte des témoins de Jéhovah de Lisieux et M. D demandent au juge des référés :

1) de suspendre l'exécution de la décision du 15 mars 2005 par laquelle le directeur de l'hôpital local d'Orbec a interdit aux membres de l'Association pour le culte des témoins de Jéhovah de rendre toute visite à M. P ;

2) de condamner l'hôpital local d'Orbec à leur verser une somme qui ne saurait être inférieure à 1500 euros sur le fondement de l'article L. 761-1 du code de justice administrative.

Ils soutiennent que M. P, âgé de 78 ans, résident depuis un an environ de la maison de retraite de l'hôpital local d'Orbec, a demandé qu'un ministre du culte de l'Association des témoins de Jéhovah lui rende visite chaque semaine afin de lui apporter une assistance spirituelle; qu'en conséquence, M. François D, membre de l'Association locale pour le culte des témoins de Jéhovah de Lisieux, a été chargé d'apporter cette assistance spirituelle à M. P qu'il rencontrait chaque samedi après-midi pendant une heure environ; que par une décision en date du 15 mars 2005, le directeur de l'hôpital local d'Orbec a interdit d'une manière générale, absolue et définitive, à toute personne appartenant à la confession des témoins de Jéhovah de rendre visite à $\mathrm{M}$. $\mathrm{P}$; que cette décision est illégale et porte gravement atteinte à certains de leurs droits fondamentaux ainsi qu'à ceux de M. P qui, par un courrier en date du 2 avril 2005, a manifesté sa volonté de voir sa liberté de culte et ses choix religieux respectés; que la condition d'urgence prévue par l'article L. 521-1 du code de justice administrative est satisfaite; qu'en effet, M. P, physiquement malade et dans la dernière période de sa vie, a vu cesser 
toute possibilité de pratiquer son culte hebdomadaire et de recevoir l'assistance spirituelle qu'il réclame ; qu'il risque de faire l'objet de pressions en vue de l'empêcher de pratiquer son culte ou de voir son libre consentement faire l'objet d'une atteinte sous forme d'administration de sédatifs ou de harcèlement; que les visites à M. P ne dépassent pas une heure par semaine et se limitent à des conversations et prières; que l'hôpital local d'Orbec n'établit pas que la mise en œuvre de conversations religieuses constituerait un danger; que l'interdiction qui procède de la décision du 15 mars 2005 porte atteinte à la liberté fondamentale qu'est la liberté de culte tant pour M. P que pour eux-mêmes, cette liberté étant garantie par l'article 9 de la Convention européenne de sauvegarde des droits de l'homme et des libertés fondamentales; que cette décision, motivée uniquement par la seule appartenance des témoins de Jéhovah à un mouvement qualifié de "sectaire ", est dépourvue de base légale dès lors qu'une telle qualification ne saurait résulter d'un rapport parlementaire; que les associations nationale et locales des témoins de Jéhovah sont des associations cultuelles et que leurs activités ne portent pas atteinte à l'ordre public; que cette décision porte atteinte aux principes de laïcité et de libre exercice des cultes et méconnaît les articles 9, 10 et 14 de la Convention européenne de sauvegarde des droits de l'homme et des libertés fondamentales, l'article 10 de la Déclaration des droits de l'homme et du citoyen ainsi que la Charte du patient hospitalisé annexée à la circulaire ministérielle $\mathrm{n}^{\circ}$ 95-22 du 6 mai 1995; que cette décision est susceptible de constituer une infraction aux articles 4327 et 225-1 du code pénal et à l'article 31 de la loi du 9 décembre 1905; que les droits fondamentaux attachés à la liberté de culte et ses corollaires ayant une valeur législative et se rattachant à des principes constitutionnels, la gravité de l'atteinte à ces principes est patente; que cette gravité ressort également de la qualification d'infraction s'attachant à une telle atteinte; que la décision susmentionnée, fondée sur une appréciation in abstracto, entraîne une rupture d'égalité entre les confessions; qu'ils n'ont jamais effectué une quelconque démarche de prosélytisme à l'intérieur de l'hôpital local d'Orbec; que le placement de M. P sous tutelle n'est pas, par lui-même, incompatible avec l'exercice de la religion de son choix; que la circulaire du 17 juillet 1978 relative aux horaires de visites aux malades hospitalisés dans les hôpitaux publics impose le droit pour le majeur protégé de recevoir des visites lorsqu'il est placé en institution; que le médecin ne peut prononcer l'interdiction totale de visites tandis que le directeur de l'établissement d'accueil ne peut que réglementer les conditions et horaires selon lesquels sont organisées les visites et que le tuteur doit respecter la volonté du majeur protégé.

Vu le mémoire en défense enregistré le 25 avril 2005, présenté pour l'hôpital local d'Orbec, représenté par son directeur, par Maître Rodolphe Rayssac, avocat; il conclut :

1) au rejet de la requête, à titre principal, comme irrecevable, à titre subsidiaire, comme non fondée ;

2) à la condamnation des requérants à lui verser la somme de 1000 euros au titre de l'article L. 761-1 du code de justice administrative.
Il soutient que les requérants ne disposent pas d'un intérêt à agir dès lors que la liberté fondamentale prétendument menacée concerne M. P et qu'ils ne démontrent pas qu'ils ont un intérêt à agir au soutien des intérêts de ce dernier; que les requérants ne démontrent pas qu'une requête à fin d'annulation aurait été enregistrée au greffe préalablement à l'introduction de la présente requête; que la condition d'urgence n'est pas satisfaite; qu'en effet, la demande de suspension n'a été enregistrée au greffe que le 15 avril 2005 alors que la décision contestée est en date du 15 mars précédent; que par une ordonnance en date du 14 avril 2005 , le juge du référé-liberté a retenu l'absence d'urgence; que si la décision contestée a pour objet d'interdire la venue des membres de l'Association des témoins de Jéhovah, M. P reste libre d'exercer la liberté de culte; que la mesure d'interdiction présente, compte tenu de son état de santé et de sa situation psychologique particulière, le caractère d'une mesure de protection de la personne de M. P, aux intérêts duquel elle ne porte pas atteinte; qu'aucune illégalité n'entache la décision du 15 mars 2005 ; qu'en effet, le directeur de l'hôpital local d'Orbec est fondé à prendre une décision interdisant l'accès des tiers à l'établissement dès lors que les requérants ne bénéficient d'aucune autorisation écrite à cette fin, alors que les dispositions de la Charte du patient hospitalisé annexée à la circulaire ministérielle du 6 mai 1995 précisent que l'accès des tiers auprès des patients ne peut avoir lieu qu'avec l'accord exprès de ceux-ci et sous réserve de l'autorisation écrite donnée par le directeur de l'établissement; qu'en application de la circulaire ministérielle du 4 octobre 2004, les associations qui organisent l'intervention de bénévoles dans les établissements de santé publics ou privés doivent conclure avec les établissements concernés une convention déterminant les modalités de cette intervention; que l'Association locale pour le culte des témoins de Jéhovah de Lisieux n'a jamais engagé une telle démarche; que la mesure d'interdiction, édictée en vue de garantir aux patients le respect de leur tranquillité, respecte les dispositions de l'article R. 111-47 du code de la santé publique; que la mesure a pour objet, compte tenu de son état physique et psychique précaire, d'assurer la protection de M. P qui est placé sous tutelle auprès du gérant de tutelle de l'hôpital local, contre les convictions notoires des témoins de Jéhovah en matière de soins; que la décision contestée respecte les principes dégagés par la jurisprudence administrative et européenne; que les risques d'altération de l'état d'une personne sont prévenus par l'article 223-15-2 du code pénal.

Vu le mémoire en réplique, enregistré le 25 avril 2005, par lequel l'Association locale pour le culte des témoins de Jéhovah de Lisieux et M. François D concluent aux mêmes fins que leur requête, par les mêmes moyens.

Ils soutiennent, en outre, que l'hôpital local d'Orbec n'établit pas en quoi l'enseignement des témoins de Jéhovah, compte tenu des conditions dans lesquelles les visites sont mises en œuvre, serait dangereux pour M. P ; qu'ils disposent d'un intérêt à agir dès lors qu'ils se voient privés de la liberté d'enseigner leur religion et que M. P est parallèlement empêché de pratiquer le culte de son choix; 
que par une requête enregistrée au greffe le 15 avril 2005, dès le lendemain de l'ordonnance du juge du référé-liberté, ils ont demandé l'annulation de la décision en date du 15 mars 2005; que le moyen tiré de ce que M. P reste libre de pratiquer le culte de son choix manque en fait dès lors que les offices célébrés à l'hôpital local d'Orbec ne relèvent pas de la religion des témoins de Jéhovah; que l'hôpital local d'Orbec ne produit aucune pièce justifiant que l'état de santé de M. P justifierait l'interdiction générale et absolue de toute visite de $\mathrm{M}$. D; que la référence à la Charte du patient hospitalisé est inopérante dès lors que l'autorisation écrite du directeur de l'hôpital n'est exigée qu'en ce qui concerne l'accès des journalistes et démarcheurs; que la référence à la circulaire ministérielle du 4 octobre 2004 relative aux conditions d'intervention de bénévoles est également inopérante; que les agissements des membres de l'Association des témoins de Jéhovah, qui est dépourvue de caractère sectaire, ne relèvent pas du champ d'application de l'article 223-15-2 du code pénal.

$\mathrm{Vu}$ la décision en litige, les autres pièces produites et jointes au dossier, le code de l'action sociale et des familes, le code de santé publique, la Constitution du 4 octobre 1958, la Déclaration des droits de l'homme et du citoyen du 26 août 1789, la Convention européenne de sauvegarde des droits de l'homme et des libertés fondamentales, la loi du 9 décembre 1905 concernant la séparation des Églises et de l'État, le code de justice administrative.

$\mathrm{Vu}$ la requête au fond $\mathrm{n}^{\circ} 0500913$ par laquelle l'Association locale pour le culte des témoins de Jéhovah de Lisieux et M. D demandent l'annulation de la décision susvisée du 15 mars 2005.

Après avoir régulièrement convoqué à l'audience publique du 26 avril 2005, à 10 heures 45 :

- l'Association locale pour le culte des témoins de Jéhovah de Lisieux, M. D et leur conseil ;

- l'hôpital local d'Orbec et son conseil.

Vu le procès-verbal de l'audience publique du 26 avril 2005 au cours de laquelle ont été entendus :

- le rapport de M. Heu, vice-président, juge des référés ;

- les observations de Maître Kaam, avocat au barreau de Paris, pour l'Association locale pour le culte des témoins de Jéhovah de Lisieux et M. D, et de M. D, requérant ;

- et les observations de M. Quillien, directeur de l'hôpital local d'Orbec.

Après avoir prononcé, à l'issue de l'audience publique à 11 heures 35 , la clôture de l'instruction.

Considérant qu'aux termes de l'article L. 521-1 du code de justice administrative: «Quand une décision administrative, même de rejet, fait l'objet d'une requête en annulation ou en réformation, le juge des référés, saisi d'une demande en ce sens, peut ordonner la suspension de l'exécution de cette décision, ou de certains de ses effets, lorsque l'urgence le justifie et qu'il est fait état d'un moyen propre à créer, en l'état de l'instruction, un doute sérieux quant à la légalité de la décision [...] »; et qu'aux termes de l'article L. 522-1 du même code: «Le juge des référés statue au terme d'une procédure contradictoire écrite ou orale. Lorsqu'il lui est demandé de prononcer les mesures visées aux articles L. 521-1 et L. 521-2, de les modifier ou d'y mettre fin, il informe sans délai les parties de la date et de l'heure de l'audience publique [...]».

\section{Sur les fins de non-recevoir soulevées par l'hôpital local d'Orbec}

Considérant, en premier lieu, que par une requête enregistrée au greffe le 15 avril 2005 sous le $n^{0} 0500913$, l'Association locale pour le culte des témoins de Jéhovah de Lisieux et $M$. D ont demandé l'annulation pour excès de pouvoir de la décision du 15 mars 2005 par laquelle le directeur de l'hôpital local d'Orbec a interdit aux membres de l'Association pour le culte des témoins de Jéhovah de rendre toute visite à $\mathrm{M}$. $\mathrm{P}$; que la présente requête à fin de suspension de l'exécution de cette décision a également été enregistrée le 15 avril 2005 au greffe du tribunal administratif; qu'ainsi, la fin de non-recevoir tirée par l'hôpital local d'Orbec de l'absence de requête au fond ne peut qu'être rejetée comme manquant en fait; qu'il en est de même, en tout état de cause, de la fin de non-recevoir tirée de ce que la requête au fond n'aurait pas été enregistrée antérieurement à la présentation de la présente requête.

Considérant, en second lieu, que la décision susvisée du 15 mars 2005 du directeur de l'hôpital local d'Orbec a pour objet d'interdire aux membres de l'Association locale pour le culte des témoins de Jéhovah de Lisieux et, notamment, à $\mathrm{M}$. D de rendre visite à $\mathrm{M}$. $\mathrm{P}$, résident au sein de cet établissement; qu'il n'est pas contesté que M. D en tant que membre de l'Association locale pour le culte des témoins de Jéhovah de Lisieux, a été chargé par cette association, sur la demande de M. P, d'apporter une assistance spirituelle à ce dernier qu'il rencontrait chaque samedi, à raison d'une heure environ; qu'ainsi, la fin de non-recevoir tirée par l'hôpital local d'Orbec de l'absence d'intérêt à agir des requérants ne saurait davantage être accueillie.

\section{Sur les conclusions aux fins de suspension}

\section{A. En ce qui concerne la condition d'urgence}

Considérant que l'urgence justifie que soit prononcée la suspension d'un acte administratif lorsque l'exécution de celui-ci porte atteinte, de manière suffisamment grave et immédiate, à un intérêt public, à la situation du requérant ou aux intérêts qu'il entend défendre; qu'il appartient au juge des référés d'apprécier concrètement, compte tenu des justifications fournies par le requérant, si les effets de l'acte litigieux sont de nature à caractériser une urgence justifiant que, sans attendre le jugement de la requête au fond, l'exécution de la décision soit suspendue.

Considérant, d'une part, qu'il ressort des pièces du dossier que M. P, âgé de 78 ans et désormais placé sous tutelle auprès du gérant de l'hôpital local d'Orbec par ordonnance du juge des tutelles auprès du tribunal d'instance de 
Mantes-la-Jolie en date du 4 juin 1998, a été admis en mars 1998 à l'hôpital local d'Orbec; que M. P a sollicité la venue dans l'établissement au sein duquel il est hébergé d'un membre de l'Association locale pour le culte des témoins de Jéhovah de Lisieux en vue de bénéficier de ce qu'il estime être une assistance spirituelle; que l'Association locale pour le culte des témoins de Jéhovah de Lisieux a chargé M. D d'apporter à M. P cette assistance spirituelle, à raison d'une visite chaque samedi après-midi pendant une heure environ; que selon une attestation établie le 10 avril 2005 par le président de l'Association locale de Lisieux, les visites rendues par M. D à M. $\mathrm{P}$ depuis quelques mois avaient pour objet d'assurer un enseignement biblique et de procurer à ce dernier une aide spirituelle; que par un document manuscrit en date du 2 avril 2005, M. P a manifesté le souhait de continuer à recevoir les visites du représentant de cette association; que, toutefois, la décision du 15 mars 2005, ayant pour objet d'interdire toute visite à $\mathrm{M}$. $\mathrm{P}$ des représentants de l'Association locale des témoins de Jéhovah, empêche, en conséquence, tout entretien sur des thèmes religieux ou spirituels entre celui-ci et le représentant de cette association et fait obstacle à la demande de M. P tendant à recevoir l'assistance spirituelle qu'il réclame au représentant de cette association.

Considérant, d'autre part, que si, pour démontrer l'absence d'urgence, l'hôpital local d'Orbec se prévaut du délai écoulé entre la décision, datée du 15 mars 2005, et l'introduction, le 15 avril 2005, de la présente requête, il n'y est, en tout état de cause, pas fondé dès lors que l'Association locale pour le culte des témoins de Jéhovah de Lisieux et M. D avaient, dès le 12 avril 2005, suite à la confirmation par M. P le 2 avril 2005 de son souhait de bénéficier d'une assistance spirituelle par un représentant de cette association, saisi le juge du référé d'une demande, présentée sur le fondement de l'article L. 521-2 du code de justice administrative, et qu'aucun élément de l'instruction ne permet d'ailleurs d'établir la date de notification de cette décision.

Considérant, enfin, que si l'hôpital local d'Orbec soutient que la mesure d'interdiction de toute visite édictée par la décision du 15 mars 2005 a été prise en vue de protéger la personne de M. P, il ne démontre pas - quand bien même il est vrai que l'intéressé est placé, du fait de sa fragilité mentale et / ou psychique, sous tutelle auprès du gérant de l'hôpital local d'Orbec - que les visites du représentant de l'Association locale pour le culte des témoins de Jéhovah de Lisieux, d'ailleurs jusqu'alors organisées à raison d'un entretien hebdomadaire d'une heure seulement, constitueraient un danger pour celui-ci ou compromettraient sa santé; que si, dans ses observations orales, le directeur de l'hôpital local d'Orbec précise que M. P a présenté une demande tendant à rencontrer la personne responsable du service religieux, cette circonstance n'est pas de nature à faire obstacle à ce que, parallèlement, il reçoive les visites d'un représentant de l'Association locale des témoins de Jéhovah.

Considérant qu'il résulte de tout ce qui précède que l'exécution de la décision en litige doit, dans les circonstances de l'espèce, être tenue comme portant une atteinte grave et immédiate à la situation de l'Association locale pour le culte des témoins de Jéhovah de Lisieux et de M. D et aux intérêts qu'ils défendent; que, par suite, la condition d'urgence, fixée à l'article L. 521-1 précité du code de justice administrative, doit être regardée comme remplie.

\section{B. En ce qui concerne la condition tenant à l'existence d'un doute sérieux}

Considérant que le directeur de l'hôpital local d'Orbec a motivé sa décision du 15 mars 2005, interdisant les visites à $\mathrm{M}$. $\mathrm{P}$ des membres de l'Association locale des témoins de Jéhovah, par l'état de santé de celui-ci et le fait que l'association serait « officiellement reconnue comme appartenant aux mouvements sectaires »; que, d'une part, le seul fait que l'Association des témoins de Jéhovah ait été reconnue dans un rapport parlementaire comme étant au nombre des mouvements sectaires est, par lui-même, sans incidence sur l'appréciation des circonstances de l'espèce et de la nécessité qui s'attacherait pour $\mathrm{M}$. $\mathrm{P}$, compte tenu de sa fragilité psychique, à voir définitivement interdit tout entretien d'ordre spirituel avec M. D, membre de l'Association locale pour le culte des témoins de Jéhovah de Lisieux; que l'hôpital local n'apporte aucun élément concret de nature à établir le bien-fondé d'une telle mesure; que, d'autre part, contrairement à ce que soutient l'hôpital local, la mesure édictée par la décision du 15 mars 2005 doit être tenue comme présentant le caractère d'une interdiction générale et absolue, nonobstant le fait que M. P puisse participer aux activités religieuses organisées au sein de l'établissement, dès lors que cette décision prive les requérants ainsi que $\mathrm{M}$. $\mathrm{P}$ de toute possibilité de se rencontrer en vue d'aborder des thèmes religieux ou spirituels; qu'ainsi, le moyen tiré par l'Association locale pour le culte des témoins de Jéhovah de Lisieux et M. D de ce que la décision contestée revêt le caractère d'une interdiction générale et absolue au droit des requérants d'aborder avec $\mathrm{M}$. $\mathrm{P}$ des thèmes religieux ou spirituels, non justifiée par la nécessité d'assurer la protection de celui-ci, est propre à créer, en l'état de l'instruction, un doute sérieux quant à la légalité de cette décision.

Considérant qu'il résulte de tout ce qui précède qu'il y a lieu de suspendre l'exécution de la décision du 15 mars 2005 par laquelle le directeur de l'hôpital local d'Orbec a interdit aux membres de l'Association pour le culte des témoins de Jéhovah de rendre toute visite à M. P.

\section{Sur les conclusions tendant à l'application de l'article L. 761-1 du code de justice administrative}

Considérant qu'aux termes de l'article L. 761-1 du code de justice administrative : «Dans toutes les instances, le juge condamne la partie tenue aux dépens ou, à défaut, la partie perdante, à payer à l'autre partie la somme qu'il détermine, au titre des frais exposés et non compris dans les dépens. Le juge tient compte de l'équité ou de la 
situation économique de la partie condamnée. Il peut, même d'office, pour des raisons tirées des mêmes considérations, dire qu'il n'y a pas lieu à cette condamnation. »

Considérant, d'une part, qu'il n'y a pas lieu, dans les circonstances de l'espèce, de faire droit à la demande de frais irrépétibles présentée dans cette instance par l'Association locale pour le culte des témoins de Jéhovah de Lisieux et M. D.

Considérant, d'autre part, qu'en vertu des dispositions précitées de l'article L. 761-1 du code de justice administrative, le tribunal ne peut pas faire bénéficier la partie perdante du paiement par l'autre partie des frais qu'elle a exposés à l'occasion du litige soumis au juge; que, dès lors, les conclusions présentées à ce titre par l'hôpital local d'Orbec ne peuvent qu'être rejetées.

\section{Ordonne :}

Article $1^{\text {er }}$ : l'exécution de la décision du 15 mars 2005 par laquelle le directeur de l'hôpital local d'Orbec a interdit aux membres de l'Association pour le culte des témoins de Jéhovah de rendre toute visite à M. P, est suspendue.

Article 2 : les demandes de frais irrépétibles présentées, d'une part, par l'Association locale pour le culte des témoins de Jéhovah de Lisieux et M. D, d'autre part, par l'hôpital local d'Orbec sont rejetées.

\section{Note}

L'ordonnance ci-dessus, devenue définitive, mérite d'être publiée dans son texte intégral contrairement aux pratiques éditoriales qui habituellement ne reproduisent pas, ou seulement par extrait ou synthèse, les visas des décisions de justice. En effet, outre l'occasion que fournit cette ordonnance d'illustrer concrètement les mécanismes de garantie juridictionnelle de la liberté de religion, le rapprochement des moyens soutenus par les requérants, tels qu'ils sont analysés dans les visas, et des motifs retenus par le juge des référés pour fonder la mesure de suspension ordonnée permet d'éclairer le raisonnement tenu en l'espèce, qui ne va pas de soi.

Âgé de 78 ans, M. Michel P a été placé sous tutelle en 1998 ; il est résident de la maison de retraite gérée par l'hôpital local d'Orbec-en-Auge (Calvados) où il recevait jusqu'à présent la visite hebdomadaire d'un membre de l'Association locale pour le culte des témoins de Jéhovah de Lisieux, M. François D. Ces visites sont présentées par l'association comme une assistance spirituelle d'une durée d'environ une heure. Par décision en date du 15 mars 2005, le directeur de l'hôpital a définitivement interdit ces visites; ce n'est que le 12 avril que l'association et M. D ont saisi le juge des référés du Tribunal administratif de Caen sur le fondement des dispositions de l'article L. 521-2 du code de justice administrative. Statuant le 14 avril 2005 sur la demande tendant à ce qu'il soit enjoint à l'établissement hospitalier de laisser les membres de l'association rendre visite à $\mathrm{M}$. $\mathrm{P}$, le juge du référé-liberté a estimé qu’il ne résultait pas de l'instruction « qu'une mesure visant à sauvegarder une liberté fondamentale doive être prise dans les quarante-huit heures ${ }^{1}$. L'ordonnance reprend ainsi la jurisprudence désormais bien établie en matière de référéliberté, qui place très haut l'exigence relative à la condition d'urgence et qui prend notamment en considération la rapidité avec laquelle le recours a été présenté; il doit être justifié d'une urgence toute particulière, allant au-delà de la condition requise en matière de référé-suspension. Par cette première ordonnance, le juge du référé-liberté a donc rejeté la requête tout en indiquant de manière explicite aux requérants qu'il leur appartenait, s'ils s'y croyaient fondés, de déposer à l'encontre de la décision attaquée un recours en annulation voire également une demande de suspension sur le fondement de l'article L. 521-1 du code de justice administrative; cette indication ${ }^{2}$ constitue aussi un fondement de la décision de rejet de la demande de référéliberté, l'existence d'autres voies de droit adéquates confortant la solution. C'est dans ces conditions qu'un recours pour excès de pouvoir et une demande de suspension ont été présentés devant le même tribunal par l'Association locale pour le culte des témoins de Jéhovah de Lisieux et M. D.

Au terme de la seconde procédure, l'ordonnance de référé du 26 avril 2005 reproduite ci-dessus prononce la suspension de la décision d'interdiction de visite jusqu'au jugement du recours en annulation. Il est inutile de s'arrêter sur toutes les questions juridiques tranchées par cette ordonnance, notamment sur les fins de non-recevoir. On observera essentiellement que, si cette affaire révèle de la part de l'administration une attitude maladroite, la démarche empruntée par le juge administratif n'est pas non plus dépourvue de toute ambiguïté.

\section{La maladresse de l'administration}

La décision attaquée est malhabile à deux égards. Mais il ne faut pas s'y tromper ; l'administration ne peut être regardée en l'espèce comme un gigantesque monstre froid qui, sciemment, chercherait à porter atteinte à la liberté de religion. D'une part, la décision attaquée a été prise par un établissement sanitaire et social de petite dimension et disposant de peu de moyens matériels et humains ${ }^{3}$. D'autre part, l'intention de l'autorité administrative est, à l'évidence, étrangère à cette volonté politique qui s'est traduite dans le passé par des mesures vexatoires $^{4}$; notre époque n’est plus marquée par un laïcisme

1. TA Caen 14 avril 2005, M. François D et Association locale pour le culte des témoins de Jéhovah de Lisieux, AJDA, $2005, \mathrm{p} .1255$.

2. Indication que l'on retrouve dans la jurisprudence : CE 9 août 2004, M. et $\mathrm{M}^{\mathrm{me}}$ Yilmaz, AJDA, 2004 p. 2414.

3. Il n'est pas indifférent que l'hôpital, bien que partie perdante, n'ait pas été condamné aux frais irrépétibles.

4. Par exemple, cet ordre donné par un maire de déposer dans l'église le cadavre d'un suicidé par noyade : CE sect. 9 janvier 1931, Abbé Cadel, Lebon p. 11,

S., 1931.3.41, note Bonnard; ou cette délibération de conseil municipal autorisant l'installation d'un urinoir public contre une église : TC 4 juillet 1934, Curé de Réalmont, Lebon p. 1247. 
militant ${ }^{5}$ et, même si parfois des pratiques regrettables peuvent encore être relevées ${ }^{6}$, celles-ci résultent d'un zèle plus maladroit que délibéré. En réalité, la décision attaquée s'avère particulièrement expédiente; le directeur de l'hôpital local a, en effet, écrit le 15 mars 2005 à l'Association locale pour le culte des témoins de Jéhovah de Lisieux le courrier suivant:

Mon attention a été attirée sur les visites de membres de votre association à M. Michel P résident de la maison de retraite de l'Hôpital local d'Orbec. Votre association est officiellement reconnue comme appartenant aux mouvements sectaires. L'état de santé de M. P nécessite qu'il soit protégé. En conséquence et en application de la réglementation actuellement en vigueur l'accès de l'établissement est formellement interdit à tous les représentants des sectes. J'ai informé, ce jour, M. P que je vous interdit: - de rendre visite à M. P - l'accès de l'établissement pour toutes autres personnes qui y résident. Je ne manquerai pas de donner les suites nécessaires si vous ne faites pas respecter cette interdiction.

On le voit, la décision litigieuse ne relève pas d'un raisonnement juridique bien argumenté; piètre application de la théorie des actes administratifs, elle est représentative de ces multiples mesures quotidiennes dont le contentieux ne rend compte que de manière aléatoire. Toutefois, quelles que soient les qualités juridiques de la décision, la démarche de l'autorité administrative se fonde sur la protection du patient; elle estime que ce dernier se trouve mis en danger par sa fréquentation des témoins de Jéhovah, compte tenu de son âge et de sa fragilité impliquant qu'il soit placé sous un régime de tutelle. Mais, indépendamment de ces bonnes intentions, l'administration commet une double maladresse.

D'abord, la décision reflète un certain et fréquent malaise à l'égard des organisations appelées « sectes». Pourtant il ne devrait pas être nécessaire de rappeler que, dans un État laïque, le droit respecte le principe de neutralité même à l'égard de ces organisations ${ }^{7}$; notamment, l'appartenance à une secte n'est pas en elle-même un obstacle à l'entrée dans la fonction publique ${ }^{8}$. Mais le principe de neutralité n'interdit pas pour autant aux pouvoirs publics de mener diverses actions, "eu égard aux risques que peuvent présenter les pratiques de certains organismes communément appelés sectes, alors même que ces mouvements prétendent également poursuivre un but reli- gieux ${ }^{9}$. Sous réserve des mesures de contrôle ou de répression appelées par les dérives sectaires, le principe de neutralité de l'État interdit toute discrimination à raison du seul contenu d'une croyance. Le rapprochement pourra d'ailleurs être fait avec les opinions politiques ; même dans le contexte de la guerre froide, l'adhésion au communisme n'a pas justifié en France, à la différence des législations d'autres démocraties occidentales, l'exclusion de la fonction publique ${ }^{10}$.

Ensuite, l'administration se comporte maladroitement à l'égard de l'état de santé de M. P, invoqué comme exigeant une protection particulière qui se manifeste par la mesure de tutelle dont il a fait l'objet. Une telle protection du patient contre lui-même est parfois admise ${ }^{11}$; mais, au cas particulier, il est jugé que la seule circonstance que M. P soit placé sous tutelle ne suffit pas à démontrer que les visites de M. D, limitées à la durée d'une heure, constitueraient un danger pour la personne âgée. On peut penser que la production de certificats médicaux détaillés, attestant des perturbations éventuellement entraînées par ces visites, aurait pu inverser la solution. L'administration ne saurait demander au juge être crue sur parole et un minimum d'éléments justificatifs était nécessaire pour qu'elle soit regardée en l'espèce comme démontrant le danger allégué. La position de l'administration n'est donc pas écartée par principe ; le raisonnement du juge est incontestable.

Mais, sur d'autres points, la lecture de l'ordonnance de référé laisse plus dubitatif.

\section{L'ambiguïté du juge administratif}

L'ordonnance de référé apparaît sans surprise en ce qui concerne la condition tenant à l'urgence. Mais, s'agissant de la seconde condition susceptible de permettre la suspension de la décision critiquée, c'est-à-dire l'existence d'un moyen propre à créer un doute sérieux, cette ordonnance procède d'une confusion, se fonde sur un motif inadéquat et occulte une liberté fondamentale. Toutefois, il convient de rappeler d'emblée combien il est délicat pour le juge de l'urgence, statuant seul, au terme d'une procédure sommaire, et orale éventuellement, de déceler, dans un fatras de faits et de moyens, le fondement pertinent de la décision qui doit être prise.

5. Après guerre, Jean Rivero constate que le Conseil d'État doit annuler un certain nombre de décisions administratives s'avérant discriminatoires à l'égard de la religion catholique («La notion juridique de laïcité», Dalloz, 1949, chron., p. 137).

6. «Un sapin de Noël a-t-il encore sa place à l'école laïque?», Le Monde, 17 décembre 2004; « Des parents musulmans se plaignent que l'on oblige leurs enfants à manger de la viande à la cantine», Le Monde, 8 janvier 2005; «Trois associations protestantes vont porter plainte contre le maire de Montreuil. Elles l'accusent d'avoir perturbé des cultes », Le Monde, 15 mars 2005.

7. D. Latournerie, «Sectes et laïcité», RDP, $\mathrm{n}^{\circ}$ 5, 2004, p. 1327.

8. G. Klein, «La neutralité de la fonction publique à l'épreuve du sectarisme», AJFP, novembre-décembre 2004, p. 310.

9. CE 18 mai 2005, Association spirituelle de l'église de scientologie d'Île-de-France, conclusions F. Donnat, AJDA, 2005, p. 1506, s'agissant de circulaires du garde des Sceaux aux parquets les invitant à lutter contre les dérives sectaires; également : CE 17 février 1992, Église de scientologie de Paris, Lebon p. 61, s'agissant d'une subvention de l'État à une association aux fins d'éditer une revue informant le public du danger des sectes.

10. Voir en ce sens les commentaires sous la décision du 28 mai 1954, Barel, dans Les Grands Arrêts de la jurisprudence administrative, $14{ }^{\mathrm{e}}$ éd., Paris, Dalloz, 2003 , notamment p. 478

11. CE sect. 26 octobre 2001, $\mathrm{M}^{\mathrm{me}} \mathrm{X}, A J D A, \mathrm{n}^{\mathrm{o}} 3$ / 2002, p. 259, note M. Deguergue: des médecins, pour tenter de sauver un patient se trouvant dans une situation extrême, ont pu accomplir un acte indispensable à sa survie et proportionné à son état sans commettre une faute de nature à engager la responsabilité de l'établissement hospitalier, «quelle que fût par ailleurs leur obligation de respecter sa volonté fondée sur ses convictions religieuses »; il s'agissait dans cette affaire d'un témoin de Jéhovah ayant refusé la transfusion sanguine pratiquée. 
Le motif central de l'ordonnance de suspension est ainsi rédigé : «le moyen tiré par l'Association locale pour le culte des témoins de Jéhovah de Lisieux et M. D de ce que la décision contestée revêt le caractère d'une interdiction générale et absolue au droit des requérants d'aborder avec $M$. $P$ des thèmes religieux ou spirituels, non justifiée par la nécessité d'assurer la protection de celui-ci, est propre à créer, en l'état de l'instruction, un doute sérieux quant à la légalité de cette décision.»

En premier lieu, le juge des référés se saisit ainsi d'une expression consacrée, celle d' « interdiction générale et absolue ». Or, cette formule, quasi rituelle, est utilisée par les demandeurs au stade de l'exposé des faits auquel ils procèdent en début de requête; mais un simple fait ne doit pas être confondu avec un moyen, ni même avec un moyen de fait. Le moyen se définit comme un ensemble construit d'arguments juridiques, présenté au soutient de la prétention d'une partie. En l'espèce, le juge transforme donc en moyen une simple allégation qui, il est vrai, renvoie à une jurisprudence classique mais dont la portée est souvent mal appréciée; de sorte qu'en l'espèce l'argumentation de la requête est fortement sollicitée par la confusion ainsi opérée entre faits et moyens. Il est vrai que cette pratique n'est pas inhabituelle au juge administratif ${ }^{12}$; l'interprétation des écrits procéduraux laisse aux juridictions une importante marge de manœuvre que le commentateur ne doit pas négliger.

En deuxième lieu, l'ordonnance est ambiguë en ce qu'elle retient un moyen d'illégalité qui, habituellement, est réservé aux mesures réglementaires ; en outre, la décision attaquée n'est pas véritablement générale puisqu'elle n'interdit ni les courriers ni les contacts téléphoniques.
Le raisonnement fait la part belle à l'idée courante selon laquelle une mesure de police administrative ne doit pas avoir un caractère général et absolu. Mais certains arrêts admettent cependant des décisions d'interdiction absolue à portée générale ${ }^{13}$, l'essentiel étant que leur portée soit justifiée par les circonstances de l'espèce ${ }^{14}$. Beaucoup plus que le caractère général et absolu de l'interdiction, c'est donc la proportionnalité de celle-ci qui importe pour l'appréciation de sa légalité ${ }^{15}$.

En troisième lieu, l'ordonnance fondée sur l'appréciation de la seule portée de la décision fait l'impasse sur la liberté religieuse et de culte, largement invoquée par les requérants et qui est pourtant qualifiée par le Conseil d'État de liberté fondamentale ${ }^{16}$. Certes, cette liberté doit être conciliée avec d'autres droits fondamentaux et avec les nécessités d'un bon fonctionnement du service public $^{17}$, mais en l'espèce aucun motif tiré de ces nécessités d'intérêt général ne motive la décision attaquée. Force est de constater que le juge administratif ne se place pas volontiers sur le terrain de la liberté de religion; c'est ainsi que les litiges relatifs aux refus de transfusions sanguines par les témoins de Jéhovah ${ }^{18}$ et le contentieux portant sur la location d'un stade parisien à l'Association cultuelle des témoins de Jéhovah de France ${ }^{19}$ ont été traités sur les terrains respectifs du droit au refus de soins et de la liberté de réunion, plutôt que sur le terrain du droit des convictions religieuses. De la même façon, le juge des référés se place en l'espèce sous l'angle du droit de visite des patients, indépendamment du contenu de ces visites, comme si la liberté de culte n'était pas réellement mise en cause en l'espèce. À cette réticence, deux explications peuvent être avancées. D'une part, le juge n'est pas dupe des efforts

12. Par exemple: CE 24 octobre 2001, M. Ayari, $\mathrm{n}^{\circ}$ 212634, où l'on voit le Conseil d'État, en matière de droit des étrangers, transformer une allégation de fait rédigée "en des termes succincts et maladroits» (séparation familiale) en un moyen de droit (violation de l'art. 8 de la CEDH); CE 28 mai 2003 , $\mathrm{M}^{\mathrm{me}}$ Lamande, $\mathrm{n}^{\circ} 245069$, décision par laquelle un moyen de droit est rectifié (art. 11 de l’ordonnance du 22 décembre 1958 sur le statut de la magistrature, en lieu et place de l'art. 11 de la loi du 13 juillet 1983 portant droits et obligations des fonctionnaires); et un président de section du Conseil d'État n'hésite pas à écrire que, parfois, il faut mieux que « le Conseil d'État soulève subrepticement même si ultra petita le bon raisonnement »(O. Fouquet, Rev. $a d m ., \mathrm{n}^{\circ} 323$, septembre-octobre 2001, chronique de jurisprudence fiscale, p. 488).

13. CE 13 mars 1968, Ministre de l'Intérieur c / Époux Leroy, Lebon p. 179: interdiction de l'activité des photographes-filmeurs sur toute la route nationale conduisant au Mont-Saint-Michel ainsi que sur les aires de stationnement adjacentes, pendant la saison touristique qui, seule, intéresse les professionnels en cause; CE sect. 28 novembre 1980, Commune d'Ardres, Lebon p. 449 : interdiction générale de la baignade dans un lac pour des motifs de salubrité; le fameux arrêt sur les lancers de nains valide également une interdiction générale et absolue : CE Ass. 27 octobre 1995, Commune de Morsang-sur-Orge, cf. Les Grands Arrêts de la jurisprudence administrative, $\mathrm{n}^{\circ} 104$.

14. CE 24 octobre 1986, Fédération française des sociétés de protection de la nature, Dr adm., $\mathrm{n}^{\circ} 637$ : «l'interdiction générale et absolue de toute circulation automobile sur une voie publique ne peut être légalement prononcée que pour des motifs de sécurité d'une exceptionnelle gravité »; CE 11 mars 2005, Ministre de l'Intérieur c/ Communes d'Avion et autres, AJDA, 2005 p. 1199: «les moyens tirés de ce que les mesures d'interdiction générales et absolues édictées par les arrêtés contestés ne sont pas justifiées par les troubles à l'ordre public, et notamment les risques d'incendie, invoqués par leurs auteurs est propre à créer un doute sérieux quant à leur légalité. »

15. CE 15 octobre 2004, SARL «Établissements Botti », Bull. jur. des collectivités locales, $\mathrm{n}^{\circ}$ 1/ 05, p. 25 : «eu égard aux inconvénients pour la tranquillité publique et aux risques pour la sécurité des riverains et des usagers de la voie publique résultant de la livraison de carburant sur la voie d'accès à la gare maritime, aux heures d'affluence et pendant la période estivale, le maire de Bonifacio n'a, dans les circonstances de l'espèce, porté une atteinte excessive ni à l'exercice de la liberté du commerce et de l'industrie ni à la libre concurrence. »

16. CE référé 16 février 2004, M. Ahmed B, AJDA, 2004, p. 822, note G. Guglielmi et G. Koubi.

17. «... en estimant que les nécessités du fonctionnement normal du service public faisaient obstacle à ce que M. B soit autorisé à se rendre à la mosquée chaque vendredi de 14 heures à 15 heures, alors que le règlement horaire applicable aux gardiens d'immeubles de l'office prescrit, en ce qui concerne ce jour de la semaine, une présence obligatoire de 5 heures à 8 heures, de 9 heures à 11 heures et de 14 heures à 16 heures 30 , la présidente de l'office municipal d'habitations à loyers modérés de Saint-Dizier n'a pas porté une atteinte manifestement illégale à la liberté de M. B de pratiquer la confession de son choix » (CE référé 16 février 2004 précité).

18. «Le statut de la question religieuse dans l'ensemble de ces affaires est relégué du raisonnement juridictionnel d'une manière qui intrigue, comme si le juge avait soigneusement voulu éviter cette dimension de la question» : S. Hennette-Vauchez, « Kant contre Jéhovah? Refus de soins et dignité de la personne humaine », Dalloz, 2004, $\mathrm{n}^{\circ} 44, \mathrm{p} .3155$, note 13

19. TA Paris 13 mai 2004, Association cultuelle des témoins de Jéhovah de Paris et autres, AJDA, 2004, p. 822, note G. Gonzalez; Dalloz, 2004, n 33 , p. 2398 , note A. Garay. 
diligentés devant les prétoires par divers organismes qui instrumentalisent la Justice et cherchent à se voir attribuer un «brevet de religiosité »; en évitant de caractériser une atteinte à la liberté de religion, au prix d'un raisonnement qui retient un peu artificiellement la notion d'interdiction " générale et absolue », le juge refuse d'entrer dans la distinction polémique entre secte et religion. D'autre part, si le droit ne qualifie ni la notion de religion, ni celle de culte, on peut néanmoins douter qu'une visite en tête-àtête, comme en l'occurrence, soit réellement constitutive d'une cérémonie cultuelle; d'où l'utilisation par le considérant précité de l'expression euphémique de « droit des requérants d'aborder avec $\mathrm{M}$. $\mathrm{P}$ des thèmes religieux ou spirituels».
Le juge des référés exerce un office nécessairement limité; il est juge de la seule apparence de légalité. Ainsi que le fait remarquer Emmanuel Glaser dans ses conclusions sur l'arrêt du CE 12 mai 2004, Commune de Rogerville: « un doute ou une absence de doute n'est pas une opinion, c'est un état d'esprit à un moment donné », au vu d'une instruction sommaire et à titre provisoire ${ }^{20}$. La décision litigieuse laisse effectivement la forte impression qu'une illégalité a été commise. À défaut d'éléments nouveaux versés au dossier, le juge du fond devrait confirmer l'ordonnance de référé mais, sans doute, toujours pas sur le fondement de la liberté religieuse. 